\title{
Nutritional aspects of children and adolescents with epidermolysis bullosa: literature review*
}

\author{
Ana Paula Caio Zidorio ${ }^{1}$ \\ Dryelle Oliveira Dias Leão ${ }^{1}$
}

\author{
Eliane Said Dutra ${ }^{1}$ \\ Izelda Maria Carvalho Costa
}

\begin{abstract}
Epidermolysis Bullosa is a genetic disorder that affects mainly the skin, however, all others systems are influenced. The nutritional care of children and adolescents with Epidermolysis Bullosa is a key treatment strategy, since the energy needs are increased due to the disease's metabolism, burdening the immune system and cicatrization process, symptoms caused by the disease hinder the intake and adequate absorption of nutrients, which may result in inadequate growth and development. Because this is a rare disease, there are few professionals who know the characteristics of both the clinical evolution and nutritional and dietary treatments. This literature review discusses the latest knowledge on energy and specific nutrient requirements to the dietary treatment and monitoring of children and adolescents with Epidermolysis Bullosa.
\end{abstract}

Keywords: Adolescent nutrition; Child nutrition; Epidermolysis bullosa

\section{INTRODUCTION}

The term Epidermolysis Bullosa (EB) was introduced in 1886 and refers to a group of inherited bullous diseases characterized by the appearance of blisters as a result of minimal mechanical trauma, caused by mutations in genes that encode any of the structural components of keratinocytes and the dermoepidermal junction. ${ }^{1,2}$ Mutations cause changes in proteins that are responsible for adhesion defects between cutaneous structures, leading to the formation of blisters. ${ }^{3}$ Currently EB is classified into four main groups according to the location of the skin separation, i.e., they are differentiated by the ultrastructural level at which blisters develop. Thus, they can be grouped as: epidermolytic types encompassing epidermolysis bullosa simplex (EBS) with intraepidermal separation (involved proteins: keratin 5 and 14) and junctional epidermolysis bullosa (JEB), in which separation occurs inside the lamina lucida (involved proteins: laminin 332, type XVII collagen and integrin $\alpha 634$ ); dermolytic type such as dystrophic epidermolysis bullosa (DEB) with cleavage inside the lamina densa (involved protein: type VII collagen) and a mixed type that refers to Kindler syndrome, in which separation occurs in the laminas lucida and densa (proteins involved: Type VII collagen and Kindlin I). ${ }^{2}$

EB subtypes are determined according to the mode of genetic transmission, the presence or absence of specific cutaneous and extra cutaneous findings, and also following the recommendations of the Third International Consensus on Diagnosis and Classification of EB. At least 30 different epidermolysis bullosa phenotypes have been described, each resulting from mutations in molecular genes that encode cutaneous structural proteins. ${ }^{2}$

EB is little known by professionals in general, particularly regarding the treatment and dietary and nutritional monitoring of the patients. Besides cutaneous manifestations, many EB subtypes have extracutaneous clinical features that can lead to morbidity and in some cases death. ${ }^{2}$ Therefore, its treatment requires the involvement of a multidisciplinary team trained in all the aspects that need care. Considering the lack of academic research in the area, this article is a review of the main concepts and nutritional aspects of EB treatment, in order to provide the best health care to the patient.

Received on 10.10.2013.

Approved by the Advisory Board and accepted for publication on 06.01.2014.

* Work performed at Brasília University Hospital - Brasília University (HUB-UnB) - Brasília (DF), Brazil.
Financial support: none.

Conflict of interests: none.

Universidade de Brasília (UnB) - Brasília (DF), Brazil.

(C)2015 by Anais Brasileiros de Dermatologia 


\section{METHOD}

The following electronic databases were searched: PubMed, SciELO, Lilacs and Medline, the last two through the Virtual Health Library. The search was divided into four steps:

- A survey of articles and search, in all indexes, of the keywords "Epidermolysis Bullosa / Epidermólise bolhosa", combined with the logical operator "and" "Child Nutrition" and "Adolescent Nutrition", registered in the descriptors of health sciences. The search limit activated was the chronological period between 2007 and 2013. Forty-six articles were found: 13 in PubMed, one in SciELO, 18 in Lilacs and 15 in MedLine;

- Perusal of the titles of retrieved studies, selection of articles that were potentially eligible for this review and discard of those deemed unsuitable to the objective;

- Identification of duplicated articles in the databases, and elimination of the repeated ones, reaching a total of 12 articles. Due to the small number of studies, the references cited in each article surveyed were also searched, yielding 6 additional articles with themes very akin to our research, which were also included.

- Thus we reached a total of 18 articles considered suitable for this review. Articles about children and adolescents with EB were analyzed regarding the following aspects: (1) potential extra-cutaneous manifestations that affect nutritional status, (2) nutritional depletion and needs and (3) growth.

\section{POTENTIAL EXTRACUTANEOUS CLINICAL FEATURES AFFECTING THE NUTRITIONAL STATUS OF PATIENTS WITH EB}

The gravity of EB varies from mild to severe depending on the involvement of the skin and extracutaneous manifestations, and it can be localized or generalized. Cutaneous findings may include blisters, scars, pigmentation changes, alopecia, absent or dystrophic nails, and deformity of hands and feet. ${ }^{4}$ The patient with EB, depending on its clinical type, is at risk for developing extra-cutaneous clinical symptoms. ${ }^{2}$ Eyes, teeth, oral mucosa, genitourinary, gastrointestinal, respiratory and musculoskeletal systems may all be involved. ${ }^{4}$

Gastrointestinal problems are common in different types of EB and lead to morbidity with severe nutritional deficits, resulting in refractory anemia, hypoalbuminemia, absorptive disorders and growth deficit. Complications can be observed in different parts of the gastrointestinal tract, including blistering and stenosis of the esophagus, gastroesophageal reflux disease, hiatal hernia, gastritis, peptic ulcer disease, protein-losing enteropathy, anal fissure, mega- colon, inflammatory bowel disease and constipation. Among these complications, it is worth highlighting esophageal stenosis, which limits the consistency of foods that can be ingested, so that at first there is dysphagia with solids of normal consistency, then with soft foods and eventually liquids, and without the appropriate intervention, it may ultimately hinder the swallowing of saliva. ${ }^{2}$

In a retrospective study conducted in London, including 223 children with various forms of EB treated at a national reference center, with the objective to delineate the nature and frequency of gastrointestinal disorders and identify the most common complications, it was observed that gastrointestinal problems were present in $58 \%$ of patients. In patients with EBS, constipation and gastroesophageal reflux were usually observed. In those with JEB, growth deficit and protein-losing enteropathy were the most frequently recorded events. Constipation was common in patients with DEB, requiring the use of laxatives and fiber supplements. Gastroesophageal reflux affected three quarters of children with recessive DEB; twothirds also had significant esophageal stenosis. In more than half of patients with recessive DEB, insertion of gastrostomy was required. Diarrhea affected a small but significant proportion of children with recessive $\mathrm{DEB}$, with macroscopic and/or microscopic colitis alterations in most of them. ${ }^{5}$

Constipation is one of the most common clinical features in EB patients, reported in all types of the disease and especially in recessive dystrophic EB, which occurs in $40 \%$ to $75 \%$ of cases. Constipation often occurs when defecation is painful due to perianal blisters and fissures, leading to fecal retention and increased constipation. Masticatory and swallowing difficulties result in a low-fiber diet that combined with reduced physical activity and the use of ironbased supplements, often essential to the patient with EB, can exacerbate the symptoms. Fecal impaction can result in death due to colon perforation and peritonitis. Anal stenosis, rectal prolapse, megacolon, perianal fistulas, irritable bowel syndrome, hemorrhoids and diverticular disease have also been described as clinical manifestations in patients with EB. ${ }^{2}$

Mouth and esophageal areas may also be affected in patients with EB owing to severe blister formation in the oral mucosa, followed by scarring that results in microstomia and ankyloglossia, which severely restrict food ingestion. ${ }^{6}$ Teeth may be structurally defective, often with poor quality enamel, which causes weak teeth most likely to decay, gingival diseases and extensive loss of tooth surface by erosion, also affecting food intake. ${ }^{7}$

Symptoms and signs such as weight loss, no 
weight gain for more than three months, inadequate growth, extended feeding time and vomiting may indicate decreased ability to masticate and swallow. Detailed assessment may show poor dentition, painful blisters in the mouth and/or esophagus and narrowing of the esophagus, and the interventions include dental restoration, use of corticosteroids to reduce dysphagia, esophageal dilation and feeding through gastrostomy. ${ }^{8}$

Anemia is a frequent and serious complication in severe types of EB, such as recessive DEB and junctional EB. Hemoglobin levels below $10 \mathrm{~g} / \mathrm{dl}$ difficult wound healing in patients with venous ulcers secondary to decreased tissue oxygenation. However, literature does not identify the ideal conduct to manage anemia in these cases. Appropriate cutaneous care, prevention and treatment of skin infections can minimize cutaneous blood loss. Oral iron supplementation is widely used to correct the anemia, although individual effectiveness may vary. Moreover, gastrointestinal disorders are causes for non-adherence. Transfusions should be considered in cases in which hemoglobin levels are consistently below $8 \mathrm{~g} / \mathrm{dl}$ and in symptomatic patients failing to respond to other treatments. ${ }^{9}$

\section{NUTRITIONAL COMPROMISE AND REQUIRE- MENTS IN EB}

The nutritional impairment in patients with EB is directly related to the severity of associated problems, i.e., the more severe the EB type, the more extensive the nutritional impairment. Children and adolescents with junctional or recessive dystrophic EB, as well as Dowling-Meara, a subtype of EB simplex, have a significant risk of nutritional deficits. ${ }^{10,11}$ This is due to numerous complications such as blisters and oral ulcerations, compromised esophageal mobility, esophageal stenosis, dysphagia, dental problems, digestive and absorptive disorders, anal erosions and fissures and rectal constriction. These events result in chronic constipation, blood and protein loss through the blisters, hypermetabolism secondary to the increase in thermogenesis and protein turnover, especially in the presence of infection. These issues have the greatest significance in children and adolescents due to their immense nutritional requirements to achieve proper growth. ${ }^{12}$ The interactions between these and other factors are a challenge to the establishment of the best conduct and it becomes very difficult to determine which interventions are priorities (Figure 1).

Malnutrition in EB patients, being the result of a combination of decreased food intake and increased nutrient demand, leads to failure to thrive, delayed puberty, anemia and a cascade of clinical and biological events that, together, culminate with the interrup- tion or retardation of wound healing. ${ }^{9}$ Nutritional depletion and protein-energy malnutrition can result in alteration of immunocompetence, and secondary bacterial infections have been reported in EB patients. ${ }^{6}$

The nutritional needs of children and adolescents with minimal blisters and little or no gastrointestinal involvement are unlikely to be higher than those of their healthy peers with matching age and sex. In severe forms of EB, however, it is difficult to quantify the nutritional needs due to a number of factors, such as: 1- the multisystemic, inflammatory and infectious nature of the disease; 2-variable nutritional requirements reflecting the age, extent of blisters, presence of infection, need for catch-up growth, among others; 3-difficulties associated with desirable weight gain estimates, since height is also affected by chronic inflammation, pain, osteoporosis and articular contractures and 4-difficulty in conducting clinical trials due to the limited number of patients. ${ }^{10}$

Some authors compare the energy requirements of children with EB to those of burned children, because of their increased metabolic levels and energy needs, which correspond to the affected body surface percentage. The energy requirement for burned children takes into account an equation for determining the affected body surface area and also the demands for growth maintenance and this equation can be adapted to estimate the needs of EB patients. ${ }^{6}$ A proposal, based on the equation developed for burned patients, is presented to estimate the energy require-

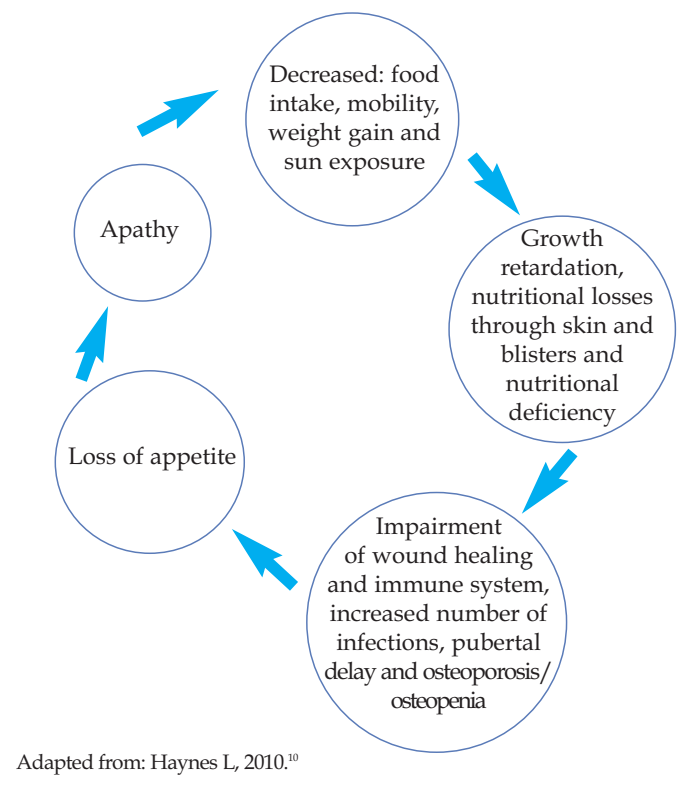

FIGURE 1: Cause-effect correlation of inadequate nutritional intake in patients with severe types of EB 
ments for children and adolescents suffering from EB, who are at nutritional risk, particularly those with junctional and dystrophic types. This equation considers a number of factors: weight, kilocalories per kilogram of body weight according to the current age at the $25^{\text {th }}$ percentile of standardized growth curves, percentage of body area affected by blisters, presence of infection and need for catch-up growth (Figure 2). This equation, despite having been released to the scientific community in 1995, is still used and cited in recent articles. ${ }^{8,10}$

However, this equation is criticized for being subjective. A more simplified method of estimating energy requirements for children and adolescents with EB is proposed, and it consists in providing 100\% to $150 \%$ of the Estimated Energy Requirement (EER), which is part of the Dietary Reference Intake (DRI). And if growth is not satisfactory, based on its regular assessments, a gradual increase in energy supply should be initiated. ${ }^{10}$ For children and adolescents who do not have nutritional risks, a likely reduction in energy consumption is suggested, to avoid the potential hazard of overweight. The latter is secondary to feet blisters that limit the activity and the ability to exercise, which decreases energy requirements and if energy consumption is not matched to its needs, weight gain and increase in pressure on the feet will occur. Therefore, energy consumption must be adjusted so that patients can reach or maintain the adequate weight for their height. ${ }^{8}$

Because of the great protein loss through blisters, the constant need for skin synthesis and recurrent infections, the protein needs of patients with EB are greater than those of their healthy peers of matching gender and age. Despite the lack of information on the quantification of cutaneous protein loss in patients with EB, it is supposed to be quite significant, which reduces the possibility of maintaining a positive nitro-

FIGURE 2: Energy requirement estimate for patients with EB

$$
\text { Energy requirement calculation }
$$

- Weight $(\mathrm{kg}) \times(\mathrm{kcal} / \mathrm{Kg}$ age corresponding to the height on the 25 th percentile) $x[1+$ (sum of 3 additional factors) $]$

- Additional factors

\begin{tabular}{|c|c|c|}
\hline $\begin{array}{l}\text { Percentage of body } \\
\text { surface with blisters }\end{array}$ & Sepsis & Catch-up growth \\
\hline $\begin{array}{l}\quad 20 \%=0.19 \\
-\quad 40 \%=0.50 \\
-100 \%=0.95\end{array}$ & $\begin{array}{l}\text { - } \quad \text { Low }=0.2 \\
\text { - } \quad \text { Moderate }=0.4 \\
\text { - }\end{array}$ & $\begin{array}{ll}- & 0.1 \\
- & 0.2\end{array}$ \\
\hline
\end{tabular}

Adapted from: Haynes L, 2007.² gen balance. ${ }^{6}$ The protein requirement is calculated using the offer of $115-200 \%$ of the estimated average requirement according to the Recommended Dietary Allowances (RDA), a component of the DRI, for the respective chronological age. ${ }^{13}$

Regarding micronutrients, there are no specific guidelines that advise on their intake for patients with EB, making the conduct, in these case, empirical. ${ }^{10}$ Micronutrient deficiencies in these patients result from losses through the skin, chronic inflammation and inadequate intake. A study including 73 patients with various types of $\mathrm{EB}$, aimed to evaluate plasmatic or erythrocyte levels of ten nutrients (vitamins A, C, B6 and B12, folate, thiamin, riboflavin, copper, zinc and iron), found low plasma levels of zinc and iron (in junctional and recessive dystrophic $\mathrm{EB}$ ), vitamin $\mathrm{C}$ (mainly EBS), vitamin A (junctional and recessive dystrophic EB), vitamin B12 (mainly EBS) and vitamin B6 (especially recessive dystrophic EB). With the exception of low iron and zinc plasma levels in JEB and recessive dystrophic $\mathrm{EB}$, only a minority of patients in any of the EB subsets had low levels of other nutrients, and an apparent correlation with malabsorption was possible only with selected nutrients. Normal levels of thiamine, riboflavin, folate and copper were found in all examined patients, regardless of EB type and the extent of cutaneous and extra-cutaneous manifestations. The authors concluded that, based on normal plasma levels of most nutrients, excessive use of vitamin supplements is unnecessary for the majority of patients with EB, especially for those with EBS and dominant DEB, in which adequate oral intake is still possible. However, considering the safe levels of daily vitamins intake, it may be a prudent practice for patients with more severe forms of JEB and recessive $\mathrm{DEB}$, since they have a more accentuated gastrointestinal involvement. ${ }^{14}$

If there are doubts adequacy intake of vitamin a specialized nutritional formula or an age-appropriated multivitamin should be recommended based on the dietary intake assessment, provided intakes does not exceed the Tolerable Upper Intake Level (UL), which is one component of DRI. Children and adolescents, who consume a significant amount of specialized nutritional formulas, acquire vitamin supplies through them. However, if the regular intake of vitamins is less than $150 \%$ of the consumption references, then a multivitamin should be prescribed. ${ }^{10}$

Despite severe lesions in grave forms of EB having similarities with thermal burns, an extrapolation of the latter's nutrient requirements should not be automatically applied to the former, because megadoses of some nutrients are supposed to be used only for a short timeframe, whereas EB patients constantly present skin lesions. In the absence of specific data 
addressing patients with EB, the development of a dietary plan regarding nutritional requirements includes: 1-Evaluation of factors that interfere with food intake and nutrient absorption; 2-Biochemical assessment, although interpretation of results may be difficult due to inflammatory response effects, which alter micronutrient concentration; 3- Estimate of nutritional intake in children and adolescents with severe forms of EB, compared with the needs of healthy individuals of same sex and age, matched height, with the addition of factors that may increase energy and protein needs. ${ }^{10}$

Regarding the causes of constipation, blisters and fissures in the anal region of patients with dystrophic and junctional EB, favor the accumulation of feces. This process stems from the fear of pain during bowel movement, moreover, other factors contribute to the problem, such as low-fiber diet, diminished fluid intake, fluid loss through skin lesions and limited physical activity. ${ }^{8}$ Although low consumption of fibers contributes to constipation in these patients, for many children an increase in the amount of fibers through conventional foods may not be appropriate or successful. These are the cases of patients with oral lesions or esophageal stenosis that difficult swallowing; those who require greater density of nutrients than is available in some kinds of foods; children younger than five years, who are unlikely to consume certain types of food in quantities enough to alleviate constipation and those with perianal lesions presenting extremely painful defecations. In such instances, the use of specialized nutritional formulas containing high percentage of insoluble fibers have been beneficial to alleviate chronic constipation. ${ }^{13}$

Feeding via gastrostomy may be necessary to improve the nutritional status of patients with more severe forms of EB. ${ }^{9}$ A retrospective study performed at the French Reference Center for Genetic Cutaneous Diseases (MAGEC), evaluated long-term enteral feeding via gastrostomy including information about energy and protein requirements, enteral feeding tolerability, growth and pubertal development in young patients with recessive DEB (REBD). It was demonstrated that the amount of calories and proteins necessary to achieve catch-up growth with enteral feeding via gastrostomy should be higher than the dietary intake references for the healthy population. The average protein intake via gastrostomy was equivalent to $180 \%$ of the dietary consumption references and the energy supply was $129 \%$. These values were expected in view of the large protein loss through the skin and its increased requirement due to the chronic inflammation observed in these patients. The authors concluded that feeding via gastrostomy should be initiated before the onset of malnutrition in order to improve growth recovery, and before the age of ten to allow pubertal development, which has a positive psychological impact. ${ }^{15}$

The goals of nutritional interventions in children and adolescents with EB are to ease the stress caused by feeding time, minimize nutritional deficiencies, improve bowel function, enhance the immunological status, optimize wound healing, build-up normal body composition and promote proper growth, as well as pubertal and sexual developments. Considering the grave prognosis of some EB types, these goals should be modified according to the patient's situation, with emphasis on the quality of life. Despite nutritional intervention, few children with other severe EB subtypes will experience significant improvement in wound healing rates, which is a disappointing, albeit not surprising fact, considering the great involvement of the skin. However the potential effect of adequate nutritional intake is more comprehensive. $^{10}$

Even if nutritional intervention is not associated with the prevention of new blisters, improvements in immunocompetence, visceral proteins and normalization of biochemical levels and, consequently, acceleration of wound healing and prevention of secondary bacterial infection have been recorded. ${ }^{6}$ The earlier the age of nutritional management start, the greater the likelihood of nutritional deficiency recovery. Although early assessment and nutritional intervention enable substantial improvements in nutritional status and growth, improve several quality of life aspects and reduce morbidity and mortality, in many cases, it is impossible to attain nutritional needs and promote satisfactory growth solely through food intake. In multisystemic conditions, such as EB, the success of nutritional intervention invariably depends on the satisfactory management of a number of associated factors.

\section{GROWTH OF CHILDREN AND ADOLESCENTS WITH EB}

Various degrees of growth retardation commonly occur in the most severely affected patients with EB, the majority has Hallopeau-Siemens recessive dystrophic EB and junctional Herlitz, with many children failing to reach the $5^{\text {th }}$ percentile of weight/height curves. ${ }^{2}$ Other authors confirmed this finding and reported that the majority of children with severe types of EB have abnormal development. ${ }^{16}$

In a study with 80 patients with different types of EB, aimed to analyze nutritional risks and growth parameters, $22 \%$ of the eighteen children with EB simplex were at risk for malnutrition, while $57 \%$ of those with junctional EB and $77 \%$ of those with dystrophic EB had nutritional risks. Height impairment, usually 
attributed to chronic protein-energy malnutrition, was observed in $11 \%$ of children with EB simplex, $29 \%$ with junctional EB and 60\% with dystrophic EB. Eighty-six percent of adults with dystrophic EB were underweight, showing that nutritional risks extend into adulthood. Among adult patients with EB simplex, $62 \%$ were overweight; probably due to reduced physical activity secondary to feet blisters. ${ }^{8}$ The study concluded that the nutritional status of patients with EB showed malnutrition with height impairment, especially in patients with junctional EB and recessive dystrophic EB.

Regarding overweight in patients with EBS, another author confirms the findings of the study aforementioned, citing that in EB simplex, excess weight is often observed in children and adolescents because lesions are mainly confined to the feet and so patients have a predominantly sedentary lifestyle and moreover, factors that in early childhood were responsible for poor oral intake of food, decrease subsequently. ${ }^{10}$

A survey was conducted with English patients and their closest peers unaffected by the disease in order to compare the birthweight of children affected by recessive EBD and a control group. It was observed that children with REBD had significantly lower birthweight, demonstrating that impaired growth begins in utero. ${ }^{17}$ Another study that did not specify EB subtypes confronted these findings affirming that, generally, children with EB are born at term with appropriate weight and height, however, after birth the disease manifestations difficult food consumption whilst nutritional needs increase. ${ }^{8}$

In the first year of life, a satisfactory growth rate can be achieved in patients with EB as result of the use of fortified infant formulas, late introduction of complementary feeding and nutrient concentration methods, although these rates are rarely sustained in severely affected children. The critical point begins during teething and at the time of the first childhood diseases, and later, when the child enters school and has less feeding periods during the day. ${ }^{13}$ A retrospective study conducted in France, including young patients with recessive DEB fed via gastrostomy, found that despite nutritional monitoring, growth retardation occurs early in the course of the disease, at an average of 3 -years for weight and 4.9 years for height. ${ }^{15}$

Special attention should be given to puberty, since this period has the highest growth rate and therefore increased nutritional demands. A child with EB presenting very low weight-to-height ratio may have less mobility and tend to rely on a wheelchair. It is important to maintain a balance between mobility, growth and nutritional status, because these are inter- related and interdependent aspects. Extremely low weight, wheelchair dependence and low bone mass are frequent in children with JEB and DREB, leading to higher probabilities of bone pain, fractures and increased immobility. Nevertheless, malnourished children may not reach puberty and benefit from the hormonal protective effects on bone health. ${ }^{10}$

Children and adolescents with more severe types of EB have significant changes in body composition, with loss of fat and muscle mass. Chronic infections are one of the causes of malnutrition and growth retardation and it is likely that the inhibitory effects of proinflammatory cytokines on growth, also observed in childhood chronic inflammatory conditions such as Crohn's disease and juvenile arthritis, can be seen in patients with severe EB, since the cytokine-mediated cascade of events leads to catabolism. ${ }^{10,16}$

Patients at the Great Ormond Street Hospital in London, with serious types of EB and growth retardation during adolescence, presented hypogonadism with low serum concentration levels of luteinizing and follicle-stimulating hormones, which are responsible for stimulating the activity of ovaries and testes. The authors also observed reduced concentrations of insulin-like growth factor-1 (IGF-1) and IGF-binding protein-3 (IGFBP3), which are proteins produced and secreted by the pituitary gland to induce the increase of cells volume and numbers, enabling tissue, organ and consequently, body growth. These same findings are observed in patients with chronic diseases. Although the hormone system has not been fully evaluated in patients with EB, malnutrition plays a major role in their growth impairment. ${ }^{16}$

The determination of the optimal growth rate for children with severe EB is complex and should always be considered in the context of the individual patient and the severity of the disease. Since complications of EB vary in number and intensity over time, it is very difficult to judge if growth and nutritional status are good. Monitoring of weight gain and growth rates are well-recognized instruments to assess the adequate development of healthy and sick children and adolescents, nevertheless, the correlation to children with EB have limited value. ${ }^{10}$

Considering the growth and development impairment of children and adolescents with EB, it is worth noting that, there is no cure for EB and that hope for those affected lays on ongoing studies in gene therapy. Meanwhile, treatments of clinical symptoms, as well as multi-professional assistance, are effective strategies just to control the disease. ${ }^{18}$

\section{FINAL CONSIDERATIONS}

Despite Epidermolysis Bullosa being a disease with cutaneous manifestations, depending on its type, 
it will present extra-cutaneous features that may affect the growth and nutritional status of children and adolescents. Among those, we should highlight anemia, constipation and other features with potential to affect food intake, oral cavity blisters, dental problems, ankyloglossia, microstomia, digestive and absorptive problems and esophageal stenosis. On the other hand, children and adolescents with EB have increased ener- gy and nutrient needs due to the growing phases, added to constant blister healing, cutaneous losses and inflammatory and infectious processes that may occur in the lesions. Therefore, the association of inadequate food intake and increased nutrient requirements leads to impaired nutritional status and consequently, overall poor health.

\section{REFERENCES}

1. Intong LR, Murrell DF. Inherited epidermolysis bullosa: new diagnostic criteria and classification. Clin Dermatol. 2012;30:70-7.

2. Fine JD, Mellerio JE. Extracutaneous manifestations and complications of inherited epidermolysis bullosa. J Am Acad Dermatol. 2009;61:387-402.

3. Oliveira ZN, Périgo AM, Fukumori LM, Aoki V. Immunological mapping in hereditary epidermolysis bullosa. An Bras Dermatol. 2010;85:856-61.

4. Bello YM, Falabella AF, Schachner LA. Management of pediatric epidermolysis bulIosa. Clin Dermatol. 2003;21:278-82.

5. Freeman EB, Köglmeier J, Martinez AE, Mellerio JE, Haynes L, Sebire NJ, et al. Gastrointestinal complications of epidermolysis bullosa in children. $\mathrm{Br} \mathrm{J}$ Dermatol. 2008;158:1308-14.

6. Gruskay DM. Nutritional management in the child with epidermolysis bullosa. Arch Dermatol. 1988;124:760-1.

7. Hubbard L, Haynes L, Sklar M, Martinez AE, Mellerio JE. The challenges of meeting nutritional requirements in children and adults with epidermolysis bullosa: proceedings of a multidisciplinary team study day. Clin Exp Dermatol. 2011;36:579-83.

8. Birge K. Nutrition management of patients with epidermolysis bullosa. J Am Diet Assoc. 1995;95:575-9.

9. Pope E, Lara-Corrales I, Mellerio J, Martinez A, Schultz G, Burrell R, et al. A consensus approach to wound care in epidermolysis bullosa. J Am Acad Dermatol. 2012;67:904-17.

10. Haynes L. Nutrition for children with epidermolysis bullosa. Dermatol Clin. 2010;28:289-301, x

11. Fedeles F, Murphy M, Rothe MJ, Grant-Kels JM. Nutrition and bullous skin diseases. Clin Dermatol. 2010;28:627-43.

12. Debra.org.uk [Internet]. Haynes L. Clinical Practice guidelines for nutrition support: in Infants and Children with Epidermolysis Bullosa (EB). Great Ormond Street Hospital. London; 2007. [cited 2012 Aug 13]. Available from: http://www.debra.org.uk/uploads/resources/EB_Guidelines_Final_for_web.pdf

13. Haynes L. Nutritional support for children with epidermolysis bullosa. J Hum Nutr Dietet. 1998;11:163-73.
14. Fine J, Tamura T, Johnson L. Blood Vitamin and Trace Metal Levels in Epidermolysis Bullosa. Arch Dermatol. 1989;125:374-9.

15. Colomb V, Bourdon-Lannoy E, Lambe C, Sauvat F, Hadj Rabia S, Teillac D, et al. Nutritional outcome in children with severe generalized recessive dystrophic epidermolysis bullosa: a short- and long-term evaluation of gastrostomy and enteral feeding. Br J Dermatol. 2012;166:354-61.

16. Martinez AE, Allgrove J, Brain C. Growth and Pubertal Delay in Patients with Epidermolysis Bullosa. Dermatol Clin. 2010;28:357-9, xii.

17. Fox AT, Alderdice F, Atherton DJ. Are children with recessive dystrophic epidermolysis bullosa of low birthweight? Pediatr Dermatol. 2003;20:303-6.

18. Boeira VL, Souza ES, Rocha Bde 0, Oliveira PD, Oliveira Mde F, Rêgo VR, et al. Inherited epidermolysis bullosa: clinical and therapeutic aspects. An Bras Dermatol. 2013;88:185-98.

\author{
MAILING ADDRESS: \\ Ana Paula Caio Zidorio \\ Hospital Universitário de Brasília \\ Unidade de nutrição clinica \\ SGAN 605, Av. L2 Norte \\ 70830-200 - Brasília - DF \\ Brazil \\ E-mail:anacaio@unb.br
}

How to cite this article: Zidório APC, Dutra ES, Costa IMC, Leão DOD. Nutritional aspects of children and adolescents with epidermolysis bullosa: literature review. 2015;90(2):217-23. 